\title{
Communication
}

\section{Exceeding the limit of dynamics studies on biomolecules using high spin-lock field strengths with a cryogenically cooled probehead}

\author{
David Ban ${ }^{\mathrm{a}}$, Alvar D. Gossert ${ }^{\mathrm{b}}$, Karin Giller ${ }^{\mathrm{a}}$, Stefan Becker ${ }^{\mathrm{a}}$, Christian Griesinger ${ }^{\mathrm{a}, *}$, Donghan Lee ${ }^{\mathrm{a}, *}$ \\ ${ }^{a}$ Dept. of NMR-based Structural Biology, Max-Planck Institute for Biophysical Chemistry, Am Fassberg 11, D-37077 Göttingen, Germany \\ ${ }^{\mathrm{b}}$ Novartis Institutes for BioMedical Research, Novartis AG, CH-4002 Basel, Switzerland
}

\section{A R T I C L E I N F O}

\section{Article history:}

Received 20 March 2012

Revised 2 May 2012

Available online 14 May 2012

\section{Keywords:}

Cryo-probe

Protein dynamics

Rotating-frame transverse relaxation

Relaxation dispersion

Ubiquitin

\begin{abstract}
A B S T R A C T
Internal motions in the microsecond timescale have been proposed to play an active part in a protein's biological function. Nuclear magnetic resonance (NMR) relaxation dispersion is a robust method sensitive to this timescale with atomic resolution. However, due to technical limitations, the observation of motions faster than $\sim 40 \mu \mathrm{s}$ for ${ }^{15} \mathrm{~N}$ nuclei was not possible. We show that with a cryogenically cooled NMR probehead, a high spin-lock field strength can be generated that is able to detect motions as fast as $25 \mu \mathrm{s}$. We apply this high spin-lock field strength in an NMR experiment used for characterizing dynamical processes. An on-resonance rotating-frame transverse relaxation experiment was implemented that allows for the detection of a $25 \mu$ s process from a dispersion curve, and transverse relaxation rates were compared at low and high spin-lock field strengths showing that at high field strengths contributions from chemical exchange with lifetimes up to $25 \mu \mathrm{s}$ can be removed. Due to the increase in sensitivity towards fast motion, relaxation dispersion for a residue that undergoes smaller chemical shift variations due to dynamics was identified. This technique reduces the previously inaccessible window between the correlation time and the relaxation dispersion window that covers four orders of magnitude by a factor of 2 .
\end{abstract}

(c) 2012 Elsevier Inc. All rights reserved.

\section{Introduction}

Internal motions in proteins are tightly related to their particular function. These motions occur in a broad range of timescales from ps to seconds and slower. NMR has been used to measure the kinetics [1-4] of such changes and the structural interconversions $[5,6]$ associated with this except for the kinetic window between the globular rotational correlation time and the fastest kinetics observed with relaxation dispersion (around $40 \mu \mathrm{s}$ for ${ }^{15} \mathrm{~N}$ nuclei). This window, depending on the correlation time of the studied protein, spans approximately four orders of magnitude. Yet, motion in this timescale has recently been proposed to play a role in molecular recognition [1,5,7]. It could be accessed only by lowering the temperature dramatically thus slowing the kinetics down that they became detectable by relaxation dispersion $[1,8]$. Therefore, we set out to expand the accessible kinetic range of relaxation dispersion as much as technically feasible.

Relaxation dispersion experiments are sensitive to motions that occur on the micro- to millisecond timescale provided that the isotropic chemical shift is modulated by this motion with a sufficient amplitude [3]. The essence of this method lies in the possibility of

\footnotetext{
* Corresponding authors. Fax: +49 5512012202.

E-mail addresses: cigr@nmr.mpibpc.mpg.de (C. Griesinger), dole@nmr.mpibpc. mpg.de (D. Lee).
}

removing the effect of the modulated isotropic shifts from the induced motion by the application of refocusing or spin-lock pulses with a certain average frequency of rotation $\left(\gamma B_{1}\right)$ [9].

In particular, rotating-frame transverse relaxation experiments using spin-lock pulses have been ideally suited to probe chemical exchange originating from motions within the microsecond timescale [9-11]. However, due to technical limitations on useable field strengths of spin-lock pulses, a time resolution for the observation of motions is restrained to processes slower than $\sim 40 \mu \mathrm{s}\left({ }^{15} \mathrm{~N} \mathrm{nu}-\right.$ clei) corresponding to $\left(\gamma B_{1}\right)^{-1}$ [12]. We have been able to surpass this limit by utilizing a cryogenically cooled NMR probehead (cryo-probehead) [13] which is a tool commonly used in many NMR facilities. We demonstrate that with a cryo-probehead high spin-lock field strengths for ${ }^{15} \mathrm{~N}$ nuclei can be safely applied and used to extend the time resolution to $25 \mu$ s and for the observation of motions that induce smaller chemical shift variances potentially allowing observation of faster motions in proteins and accurate characterization of parameters that define the dynamic process.

\section{Results}

\subsection{Measurement of spin-lock fields on a cryo-probehead}

We investigated the limit of a cryo-probehead (Bruker QCI) by measuring the highest spin-lock field strength that can be applied 
without endangering the intactness of the probehead. Off-resonance continuous wave decoupling during the acquisition time $\left(t_{2, \max }=122.1 \mathrm{~ms}\right)$ in a $\left[{ }^{15} \mathrm{~N},{ }^{1} \mathrm{H}\right]-\mathrm{HSQC}$ was performed on a $600 \mathrm{MHz}$ spectrometer [12]. We started to measure the spin-lock field strength by applying the recommended specification for a cryo-probehead (approximately $2 \mathrm{kHz}$, corresponding to a minimum life time of $80 \mu \mathrm{s}$; black) and increased the spin-lock field strength as shown in Fig. 1. The integrity of the cryo-probehead was secured by ensuring that the coil's reserve power did not drop below $5 \%$, and that the temperature of the coil did not change during the application of the spin-lock pulse. In this way, we could achieve a spin-lock field strength of $6.4 \mathrm{kHz}$ (Fig. 1; blue) probably because of the cooling of the coil. This exceeds also the recommended specification for a room-temperature probehead which is approximately $4 \mathrm{kHz}$ (Fig. 1; red) and limited to this value since the coil is not cooled [14]. The accurate extraction of the kinetics of the chemical exchange process from relaxation dispersion measurements also relies on the signal to noise of the NMR measurement. This is lower by a factor of $2-3$ in a room-temperature probe. Thus, for optimal sensitivity we have to compare the $6.4 \mathrm{kHz}$ with the so far specified value of $2 \mathrm{kHz}$, constituting a 3.2-fold increase of the field strength.

\subsection{Validation for relaxation dispersion experiments}

We then applied this high spin-lock field strength to an on-resonance rotating-frame transverse relaxation $\left(R_{1} \rho\right)$ experiment (Fig. 2) on the same cryo-probehead [15]. Since the maximal kinetic rate of an exchange process detectable by the on-resonance $R_{1 \rho}$ experiment is proportional to the applied spin-lock field strength, only motions slower than $\sim 80 \mu \mathrm{s}=(2 \pi * 2 \mathrm{kHz})^{-1}$ $\left(\sim 40 \mu \mathrm{s}=(2 \pi * 4 \mathrm{kHz})^{-1}\right)$ could be detected using the previously recommended specification of a spin-lock field for a cryo-probe-

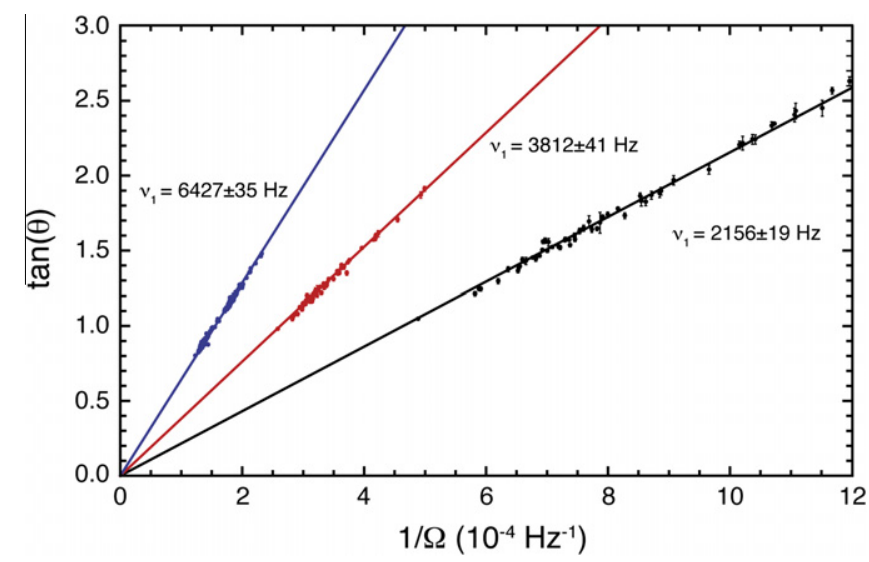

Fig. 1. Measurement of spin-lock field strengths $\left(v_{1}\right)$. Spin-lock field strengths that match with the previous technical specifications when using a cryo-probehead and a room-temperature probehead are shown in black (approximately $2 \mathrm{kHz}$ ) and red (approximately $4 \mathrm{kHz}$ ) [14]. The newly achieved high spin-lock field strength with the use of a cryo-probehead is shown in blue $(6.4 \mathrm{kHz})$. The tilt angles $(\tan (\theta))$ were calculated using an equation $\left(\sqrt{\left(J_{0} / J_{R}\right)^{2}-1}\right)$ where $J_{0}$ and $J_{R}$ are scalar coupling between amide proton and nitrogen and its reduced scalar coupling [12], respectively. $J_{R}$ were measured with off-resonance continuous wave $(\mathrm{CW})$ decoupling during the acquisition in a $\left[{ }^{15} \mathrm{~N},{ }^{1} \mathrm{H}\right]-\mathrm{HSQC}[12] . \Omega$ is the frequency difference between an observed ${ }^{15} \mathrm{~N}$ resonance and the applied $\mathrm{CW}$ decoupling. All scalar coupled ${ }^{15} \mathrm{~N}$ resonances were then fit with respect to $\Omega$ which yields a linear correlation with $v_{1}$ as the slope $\left(\tan (\theta)=v_{1} / \Omega\right.$. All experiments were conducted on a 14.1 T magnet with a Bruker QCI 600S3 cryo-probehead at $277 \mathrm{~K}$ with 1024 $\left(t_{2, \max }=122.1 \mathrm{~ms}\right)$ and $256\left(t_{1, \max }=140.3 \mathrm{~ms}\right)$ complex points in the direct and indirect dimensions, respectively. A long recycle delay was employed that maintained the duty cycle to be $2.4 \%$ for all experiments. Errors were calculated from error propagation with the measured errors in $J_{0}$ and $J_{R}$ which were evaluated based on the line width at half-height of coupled peaks and their signal-to-noise ratio. head of $2 \mathrm{kHz}$ ( $4 \mathrm{kHz}$ on a room-temperature probehead). With the newly achieved high spin-lock field strength of $6.4 \mathrm{kHz}$, fast motions around $25 \mu \mathrm{s}=(2 \pi * 6.4 \mathrm{kHz})^{-1}$ are accessible with an on-resonance $R_{1} \rho$ experiment. In order to control sample heating from different employed spin-lock field strengths a control scheme was implemented in which the same power was used in all experiments [16]. For each experiment, the length and strength of the employed spin-lock and the maximum power that would be used for the dispersion measurement at the highest spin-lock field were considered and a variable duration temperature control block was applied with the highest power at the end of acquisition for every collected transient. This scheme was effective in controlling the fluctuation in temperature when field strengths were varied as flat dispersion curves were obtained (see Fig. S1 for an example). A recycle delay was chosen that kept the duty cycle at $5 \%$.

Using a ${ }^{15} \mathrm{~N}$-labeled ubiquitin sample at $277 \mathrm{~K}$, we measured relaxation dispersion for residues Ile13, Thr55, and Val70, which have been previously reported to show dispersion $[1,17,18]$. We used an on-resonance $R_{1} \rho$ experiment [15] in which the spin-lock field strength was varied from 1 to $6 \mathrm{kHz}$ allowing for residues that undergo chemical exchange to be modulated solely by the utilized spin-lock field strength (Fig. 2). Using a fast exchange model, values for exchange lifetime $\left(\tau_{\text {ex }}\right)$ (chemical shift variance $(\Phi)$ ) of $50 \pm 9 \mu \mathrm{s}\left(29 \pm 7 \times 10^{3} \mathrm{rad}^{2} \mathrm{~s}^{-2}\right), 51 \pm 5 \mu \mathrm{s}\left(53 \pm 7 \times 10^{3} \mathrm{rad}^{2} \mathrm{~s}^{-2}\right)$, and $33 \pm 5 \mu \mathrm{s}\left(112 \pm 38 \times 10^{3} \mathrm{rad}^{2} \mathrm{~s}^{-2}\right)$ for Ile13, Thr55, and Val70, respectively, were obtained. They are in good agreement with previous studies $[1,17,18]$. When the previously recommended specification of spin-lock field strength for a cryo-probehead (up to $2 \mathrm{kHz}$; hashed box in Fig. 2) was used for fitting the dispersion data we could not achieve reliable fitting results due to the fact that the changes in the effective relaxation rate $\left(R_{2, \text { eff }}\right)$ of residues are very small $R_{2, \text { eff }}^{1 \mathrm{kHz}}-R_{2, \text { eff }}^{2 \mathrm{kHz}}=0.26,0.64$, and $0.43 \mathrm{~s}^{-1}$ for Ile13, Thr55, and Val70, respectively).

Measuring up to a spin-lock field strength specification for a room-temperature probehead ( $4 \mathrm{kHz}$; dashed line in Fig. 2), now performed on a cryo-probehead, gave changes in $R_{2, \text { eff }}$ that were

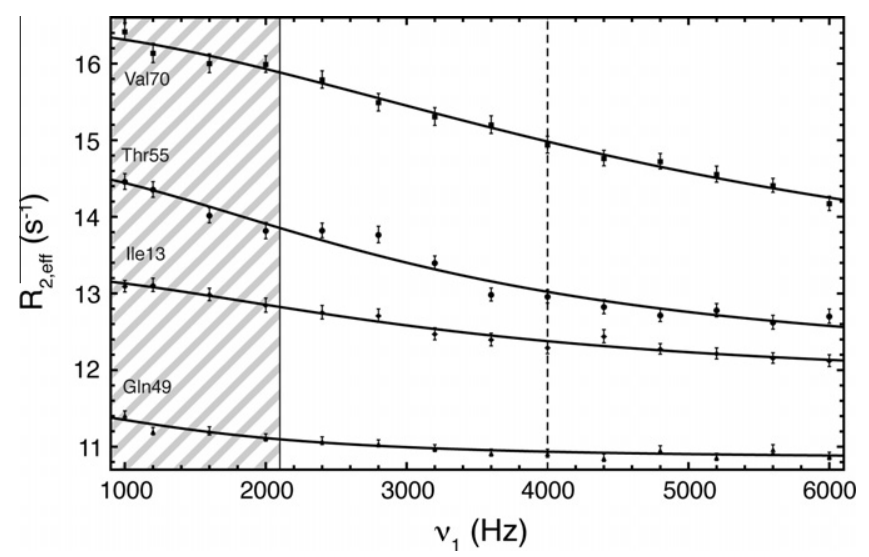

Fig. 2. Relaxation dispersion curves of residues Ile13 (diamond), Gln49 (triangle), Thr55 (circle), and Val70 (square) in ubiquitin measured with an on-resonance $R_{1 \rho}$ experiment [15]. The effective transverse relaxation rates $\left(R_{2, \text { eff }}\right)$ are plotted against spin-lock field strengths $\left(v_{1}\right)$. Previous technical specification of spin-lock field strengths of a cryo-probehead and a room-temperature probehead are indicated with solid (approximately up to $2 \mathrm{kHz}$ ) and dashed lines (approximately up to $4 \mathrm{kHz}$ ), respectively. The recorded dispersion data was fit to the fast-exchange model of $R_{2, \text { eff }}=R_{2}{ }^{0}+\Phi \tau_{\mathrm{ex}} /\left(1+\left(\omega_{1} \tau_{\mathrm{ex}}\right)^{2}\right)$ in which $\omega_{1}=\left(2 \pi v_{1}\right), R_{2}{ }^{0}$ is the intrinsic transverse relaxation rate, $\Phi$ is the population weighted chemical shift variance, and $\tau_{\mathrm{ex}}$ the lifetime of the exchange process. $R_{2, \text { eff }}$ rates were determined using a two point sampling scheme, in which a reference spectrum was recorded without a spin-lock period, and a single relaxation delay was used for all spectra where a spinlock was applied [15,32]. For each spectrum 128 scans were collected with a recycle delay of 3 seconds. The total measurement time for each dispersion curve was 1.8 hours. Errors in $R_{2, \text { eff }}$ were propagated from the noise in the reference and spinlocked spectra. 
large enough to provide fits that agree with literature values [1,17]. In order to evaluate the dependency between the quality of the fits and the field strengths, we recorded a dispersion curve of Val70 with a total of 51 spin-lock field strengths up to $6 \mathrm{kHz}$ and conducted a jackknife procedure for the fitting. Eleven field strengths up to 2, 4, and $6 \mathrm{kHz}$ were randomly selected 500 times and then subsequently fit to a fast-exchange model. As stated before, data up to $2 \mathrm{kHz}$ could not provide a reliable fit. The average values and errors from the runs yielded $\Phi$ and $\tau_{\text {ex }}$ of $163 \pm 149 \times 10^{3}$ $\operatorname{rad}^{2} \mathrm{~s}^{-2}$ and $32 \pm 10 \mu \mathrm{s}\left(101 \pm 58 \times 10^{3} \operatorname{rad}^{2} \mathrm{~s}^{-2}\right.$ and $\left.36 \pm 10 \mu \mathrm{s}\right)$ for 4 (6) $\mathrm{kHz}$. Evidently, the use of higher field strengths gives fits with lower errors.

In addition, we measured relaxation dispersion for residue Gln49, which is involved at the binding interface for ubiquitin associated interactions $[19,20]$ and has not been previously identified when spin-lock field strengths lower than $6 \mathrm{kHz}$ were utilized (Fig. 2) $[1,17,18]$. Although smaller changes in $R_{2, \text { eff }}$ are observed (an order of magnitude smaller than Ile13), it was possible to extract a $\tau_{\mathrm{ex}}$ and $\Phi$ of $101 \pm 32 \mu \mathrm{s}$ and $7.1 \pm 1.2 \times 10^{3} \mathrm{rad}^{2} \mathrm{~s}^{-2}$, respectively. Comparison of experimental $\Phi_{\mathrm{Gln} 49}$ with $\Phi_{\mathrm{Ile} 13}$ yields a ratio of $4.1 \pm 1.7$, which compares well with ratios predicted from the published ubiquitin ensembles [1,5,7] ranging from 3.8 to 1.5 (Table S1).

\subsection{Application to the accurate determination of transverse relaxation rates}

In addition to dispersion type experiments, high spin-lock field strengths can be used to quantify transverse relaxation rates $\left(R_{2}\right)$ $[11,21]$. Typically, $R_{2}$ together with longitudinal relaxation rate $\left(R_{1}\right)$ and heteronuclear NOE is used to obtain information of motions faster than the overall tumbling motion of a macromolecule (the Lipari-Szabo order parameter, $S_{L S}^{2}$ ) $[22,23]$. For this analysis, an $R_{2}$ experiment which ideally suppresses exchange contributions $\left(R_{\mathrm{ex}}\right)$ to $R_{2, \mathrm{eff}}$ is highly desirable. Residual $R_{\mathrm{ex}}$ contributions systematically disturb the analysis and the fitting procedure for $R_{2}[24,25]$. Thus, the higher spin-lock field strength would provide more accurate $R_{\text {ex }}$ free $R_{2}$ values since motions slower than $25 \mu \mathrm{s}$, as described earlier, can be refocused by using a $\sim 6 \mathrm{kHz}$ spin-lock field.

We compared $R_{2, \text { eff }}$ values with spin-lock field strengths of 1 and $6 \mathrm{kHz}$ on a ${ }^{15} \mathrm{~N}$-labeled ubiquitin sample at $277 \mathrm{~K}$. From the 72 potentially observable sites, 46 resonances provided sufficient intensity and frequency separation in either the ${ }^{1} \mathrm{H}$ or ${ }^{15} \mathrm{~N}$ dimension to be reliably queried with the employed selective on-resonance experiment (Fig. 3A) [15]. Their differences can be found in the Supplementary Material (Fig. S2). Fig. 3B shows $R_{2, \text { eff }}$ as

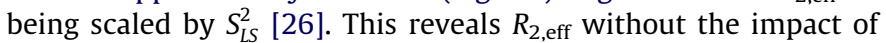
motions faster than the overall tumbling time of ubiquitin and iso-

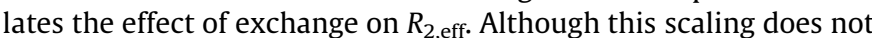
account for diffusion anisotropy, ubiquitin has only small rotational anisotropy [25] and therefore, the effect of this motion has nearly a uniform effect for all $R_{2, \text { eff }}$. The variances of $R_{2, \text { eff }} / S_{L S}^{2}$ using $6 \mathrm{kHz}\left(0.57 \mathrm{~s}^{-2}\right)$ and $1 \mathrm{kHz}\left(0.95 \mathrm{~s}^{-2}\right)$ spin-lock field strengths, show a reduction by $40 \%$ (Fig. $3 \mathrm{~B}$ ). Also, the average $R_{2, \text { eff }} / S_{L S}^{2}$ is smaller for $6 \mathrm{kHz}$ as compared to $1 \mathrm{kHz}$ as expected from the more efficient removal of exchange contributions.

\section{Discussion and conclusion}

We have shown that cryogenically cooled NMR probeheads can generate higher spin-lock field strengths than previously specified without endangering the intactness of the probehead. The newly achieved high spin-lock field strength $(\sim 6 \mathrm{kHz})$ makes chemical exchange kinetics down to $25 \mu$ s accessible using an on-resonance $R_{1} \rho$ experiment. Using ${ }^{15} \mathrm{~N}$ nuclei we could achieve a factor of two
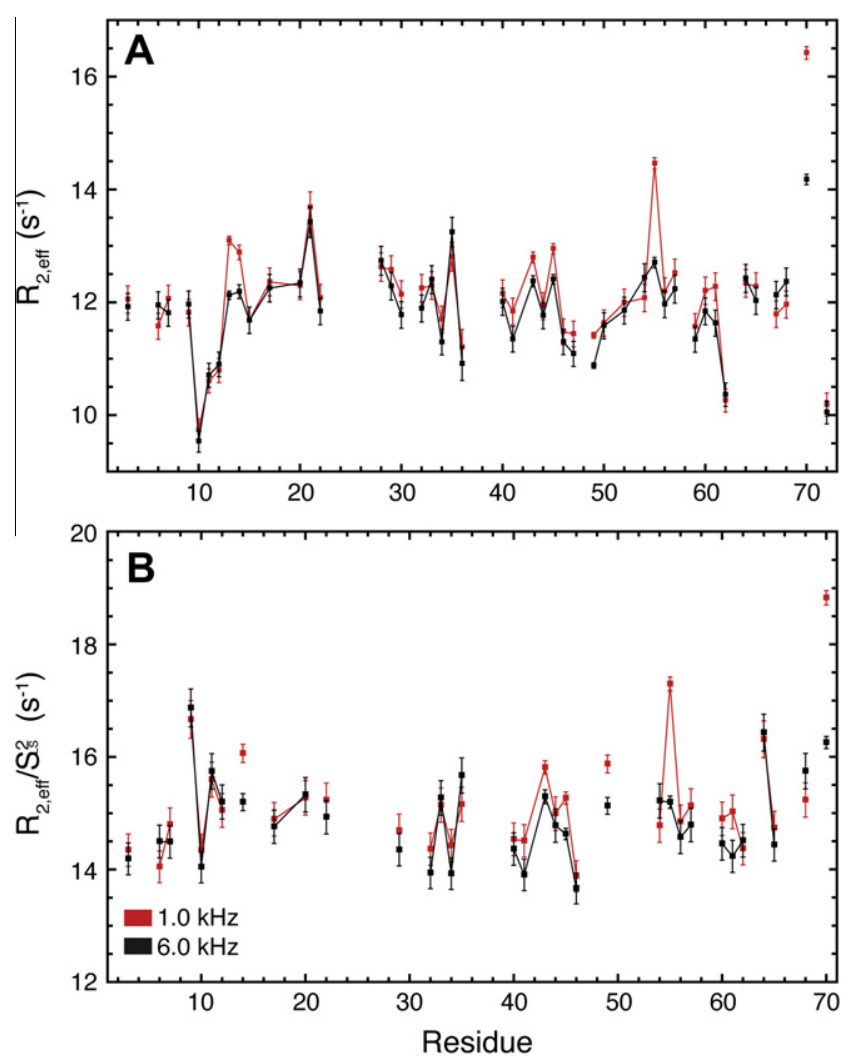

Fig. 3. Plots of effective relaxation rate $\left(R_{2, \text { eff }}=R_{2}^{0}+R_{\mathrm{ex}}\right)(\mathrm{A})$ and divided by the Lipari-Szabo order parameter $\left(S_{I S}^{2}\right)$ (B) [26]. $R_{2 \text {,eff }}$ was measured using an onresonance $R_{1} \rho$ experiment [15] for ubiquitin on a $14.1 \mathrm{~T}$ magnet at $277 \mathrm{~K}$. Errors in $R_{2, \text { eff }}$ were propagated using the protocol in Ref. [15]. Residues with determined $S_{L S}^{2}$ [25] and quantified $R_{2, \text { eff }}$ are shown for ${ }^{15} \mathrm{~N}$ sites across residues $1-72$.

improvement in the time resolution. Since sampling of the dispersion curve over larger $\gamma B_{1}$ ranges improves the analysis, motion that induces smaller chemical shift variances can be observed. This revealed dynamics in $G \ln 49$ of ubiquitin that were previously not observed.

Adapting the use of a cryo-probe for nuclei with larger gyromagnetic ratios [27-30] could also further enhance the currently set time resolution of $25 \mu \mathrm{s}$, and is our current focus for future development. In principle, off-resonance $R_{1}$ experiments can be conducted by combining large spin-lock fields and offsets which will yield even greater effective fields and thus provide dynamic information of motions even faster than $25 \mu$ s [10]. For example, an offset of $16.5 \mathrm{kHz}$ provides an effective field of $17.5 \mathrm{kHz}$ using a spin-lock field strength of $6 \mathrm{kHz}$. With this large effective field, motions slower than $9 \mu$ s could be detected. However, with this large offset, only $12 \%$ of exchange induced chemical shift variance contributes to the relaxation dispersion. Thus, the chemical shift variance induced by motions has to be large enough to observe this dynamics using off-resonance $R_{1} \rho$ experiments. The detection of small chemical shift variations could be improved by the cryoprobe detection making this off-resonance experiment especially attractive for observation of fast timescale motion. Furthermore, multiple quantum double resonance experiments, which was shown to be applicable for the studies of motions up to $50 \mu \mathrm{s}$ [18], can be used to explore further insight into dynamics that have smaller conformational amplitudes by combining the large spinlock fields demonstrated here. We have also shown that the newly introduced high spin-lock field strength allows for better refocusing of exchange processes with lifetimes up to $25 \mu$ s, thereby suppressing chemical exchange processes that are inherent to the 
transverse relaxation rate. The high spin-lock field can only be achieved with a cryo-probehead probably because of the cooling of the coil absent in normal temperature probeheads, which in addition provides a 2-3-fold sensitivity gain [31] compared to a room-temperature probehead thus making this extension to faster kinetics even more feasible.

\section{Acknowledgments}

We thank Drs. Helena Kovacs, Roberto Seydoux, and Klemens Kessler (Bruker BioSpin) for providing discussion and insight into the cryo-probehead technology. This work was supported by the Max Planck Society (to C.G.), the Fonds der Chemischen Industrie, the German Israel Funds, the DFG, the ERC (ERC grant agreement number 233227 to C.G.).

\section{Appendix A. Supplementary data}

Supplementary data associated with this article can be found, in the online version, at http://dx.doi.org/10.1016/j.jmr.2012.05.005.

\section{References}

[1] D. Ban, M. Funk, R. Gulich, D. Egger, T.M. Sabo, K.F. Walter, R.B. Fenwick, K. Giller, F. Pichierri, B.L. De Groot, O.F. Lange, H. Grubmüller, X. Salvatella, M. Wolf, A. Loidl, R. Kree, S. Becker, N.A. Lakomek, D. Lee, P. Lunkenheimer, C. Griesinger, Kinetics of conformational sampling in ubiquitin, Angew. Chem. Int. Edit. Engl. 50 (2011) 11437-11440.

[2] J. Dittmer, G. Bodenhausen, Evidence for slow motion in proteins by multiple refocusing of heteronuclear nitrogen/proton multiple quantum coherences in NMR, J. Am. Chem. Soc. 126 (2004) 1314-1315.

[3] D.F. Hansen, P. Vallurupalli, L.E. Kay, Using relaxation dispersion NMR spectroscopy to determine structures of excited, invisible protein states, J. Biomol. NMR 41 (2008) 113-120.

[4] R. Otten, J. Villali, D. Kern, F.A. Mulder, Probing microsecond timescale dynamics in proteins by methyl ${ }^{1} \mathrm{H}$ Carr-Purcell-Meiboom-Gill relaxation dispersion NMR measurements. Application to activation of the signaling protein NtrC(r), J. Am. Chem. Soc. 132 (2010) 17004-17014.

[5] O.F. Lange, N.A. Lakomek, C. Fares, G.F. Schröder, K.F. Walter, S. Becker, J. Meiler, H. Grubmüller, C. Griesinger, B.L. De Groot, Recognition dynamics up to microseconds revealed from an RDC-derived ubiquitin ensemble in solution, Science 320 (2008) 1471-1475.

[6] P. Vallurupalli, D.F. Hansen, E. Stollar, E. Meirovitch, L.E. Kay, Measurement of bond vector orientations in invisible excited states of proteins, Proc. Natl. Acad. Sci. USA 104 (2007) 18473-18477.

[7] R.B. Fenwick, S. Esteban-Martin, B. Richter, D. Lee, K.F. Walter, D. Milovanovic, S. Becker, N.A. Lakomek, C. Griesinger, X. Salvatella, Weak long-range correlated motions in a surface patch of ubiquitin involved in molecular recognition, J. Am. Chem. Soc. 133 (2011) 10336-10339.

[8] J.L. Mills, T. Szyperski, Protein dynamics in supercooled water: the search for slow motional modes, J. Biomol. NMR 23 (2002) 63-67.

[9] D.G. Davis, M.E. Perlman, R.E. London, Direct measurements of the dissociation-rate constant for inhibitor-enzyme complexes via the $\mathrm{T}_{1 \mathrm{r}}$ and $\mathrm{T}_{2}$ (CPMG) methods, J. Magn. Reson. B 104 (1994) 266-275.

[10] A.G. Palmer 3rd, F. Massi, Characterization of the dynamics of biomacromolecules using rotating-frame spin relaxation NMR spectroscopy, Chem. Rev. 106 (2006) 1700-1719.
[11] T. Szyperski, P. Luginbühl, G. Otting, P. Güntert, K. Wüthrich, Protein dynamics studied by rotating frame ${ }^{15} \mathrm{~N}$ spin relaxation times, J. Biomol. NMR 3 (1993) 151-164.

[12] A.G. Palmer 3rd, C.D. Kroenke, J.P. Loria, Nuclear magnetic resonance methods for quantifying microsecond-to-millisecond motions in biological macromolecules, Methods Enzymol. 339 (2001) 204-238.

[13] P. Styles, N.F. Soffe, C.A. Scott, D.A. Cragg, F. Row, D.J. White, P.C. White, A highresolution NMR probe in which the coil and preamplifier are cooled with liquid helium, J. Magn. Reson. 60 (1984). 497-404.

[14] J. Cavanagh, W.J. Fairbrother, A.G. Palmer 3rd, M. Rance, N.J. Skelton, Principles and Practice: Protein NMR Spectroscopy, 2nd ed., Elsevier, 2007.

[15] D.M. Korzhnev, V.Y Orekhov, L.E. Kay, Off-resonance $R_{1}$ NMR studies of exchange dynamics in proteins with low spin-lock fields: an application to a Fyn SH3 domain, J. Am. Chem. Soc. 127 (2005) 713-721.

[16] A.C. Wang, A. Bax, Minimizing the effects of radio-frequency heating in multidimensional NMR experiments, J. Biomol. NMR 3 (1993) 715-720.

[17] F. Massi, M.J. Grey, A.G. Palmer 3rd, Microsecond timescale backbone conformational dynamics in ubiquitin studied with $N M R R_{1 \mathrm{r}}$ relaxation experiments, Protein Sci. 14 (2005) 735-742.

[18] N. Salvi, S. Ulzega, F. Ferrage, G. Bodenhausen, Timescales of slow motions in ubiquitin explored by heteronuclear double resonance, J. Am. Chem. Soc. 134 (2012) 2481-2484.

[19] A. Ohno, J. Jee, K. Fujiwara, T. Tenno, N. Goda, H. Tochio, H. Kobayashi, H. Hiroaki, M. Shirakawa, Structure of the UBA domain of Dsk2p in complex with ubiquitin molecular determinants for ubiquitin recognition, Structure 13 (2005) 521-532.

[20] W.I. Sundquist, H.L. Schubert, B.N. Kelly, G.C. Hill, J.M. Holton, C.P. Hill Ubiquitin recognition by the human TSG101 protein, Mol. Cell 13 (2004) 783789.

[21] D. Long, D. Yang, Millisecond timescale dynamics of human liver fatty acid binding protein: testing of its relevance to the ligand entry process, Biophys. J. 98 (2010) 3054-3061.

[22] G. Barbato, M. Ikura, L.E. Kay, R.W. Pastor, A. Bax, Backbone dynamics of calmodulin studied by ${ }^{15} \mathrm{~N}$ relaxation using inverse detected two-dimensional NMR spectroscopy: the central helix is flexible, Biochemistry 31 (1992) 52695278.

[23] G. Lipari, A. Szabo, Model-free approach to the interpretation of nuclear magnetic resonance relaxation in macromolecules. 1 . Theory and range of validity, J. Am. Chem. Soc. 104 (1982) 4546-4559.

[24] D.M. Korzhnev, M. Billeter, A.S Arseniev, V.Y. Orekhov, NMR studies of Brownian tumbling and internal motions in proteins, Prog. Nuc. Magn. Reson. Spect. 38 (2001) 197-266.

[25] N. Tjandra, S.E. Feller, R.W. Pastor, A. Bax, Rotational diffusion anisotropy of human ubiquitin from ${ }^{15} \mathrm{~N}$ NMR relaxation, J. Am. Chem. Soc. 117 (1995) $12562-12566$

[26] S.L. Chang, N. Tjandra, Temperature dependence of protein backbone motion from carbonyl ${ }^{13} \mathrm{C}$ and amide ${ }^{15} \mathrm{~N}$ NMR relaxation, J. Magn. Reson. 174 (2005) 43-53.

[27] U. Brath, M. Akke, D. Yang, L.E. Kay, F.A. Mulder, Functional dynamics of human FKBP12 revealed by methyl ${ }^{13} \mathrm{C}$ rotating frame relaxation dispersion NMR spectroscopy, J. Am. Chem. Soc. 128 (2006) 5718-5727.

[28] C. Eichmuller, N.R. Skrynnikov, A new amide proton $\mathrm{R}_{1 \mathrm{r}}$ experiment permits accurate characterization of microsecond time-scale conformational exchange, J. Biomol. NMR 32 (2005) 281-293.

[29] R. Ishima, D.I. Freedberg, Y.X. Wang, J.M. Louis, D.A. Torchia, Flap opening and dimer-interface flexibility in the free and inhibitor-bound HIV protease, and their implications for function, Structure 7 (1999) 1047-1055.

[30] R. Ishima, P.T. Wingfield, S.J. Stahl, J.D. Kaufman, D.A. Torchia, Using amide ${ }^{1} \mathrm{H}$ and ${ }^{15} \mathrm{~N}$ transverse relaxation to detect millisecond time-scale motions in perdeuterated proteins: application to HIV-1 protease, J. Am. Chem. Soc. 120 (1998) 10534-10542.

[31] H. Kovacs, D. Moskau, M. Spraul, Cryogenically cooled probes - a leap in NMR technology, Prog. Nucl. Magn. Reson. Spect. 46 (2005) 131-155.

[32] J.A. Jones, P. Hodgkinson, A.L. Barker, P.J. Hore, Optimal sampling strategies for the measurement of spin-spin relaxation times, J. Magn. Reson. B 113 (1996) 25-34. 